\title{
On a Lamb-type problem for a bi-axially pre-stressed incompressible elastic plate
}

\author{
J. D. Kaplunov ${ }^{1}$, A. V. Pichugin ${ }^{1 *}$ and G. A. Rogerson ${ }^{2 \dagger}$ \\ ${ }^{1}$ Dept. of Mathematics, University of Manchester, \\ Oxford Road, Manchester, M13 9PL, UK. \\ 2 Dept. of Mathematics, School of Sciences, \\ University of Salford, Salford, M4 5WT, UK. \\ Dedicated to Professor Ray Ogden on his 60th birtday.
}

\begin{abstract}
The far-field response of a bi-axially pre-stressed incompressible elastic plate, subjected to an instantaneous edge impulse loading, is studied using a refined long-wave low-frequency theory. The second order correction introduced by the refined theory is demonstrated to smooth the discontinuity associated with one of the wave fronts predicted by the leading order hyperbolic theory. The character of so-called quasi-front is shown to depend greatly both on the material parameters and pre-stress and may be either classical receding or advancing. Additionally, and in contrast to the analogous problem in linear isotropic elasticity, in a pre-stressed plate the dilatational quasi-front may propagate slower than the shear wave front. This situation is demonstrated to lead to the formation of a head-wave quasi-front.
\end{abstract}

\section{Introduction}

Lamb's problem, i.e. the problem of determining the response of an isotropic elastic half-space to an instantaneous edge point loading, has become one of the classical model problems for studying non-stationery phenomena in elastic solids. The original problem, see Lamb (1904), is quite well understood now due to works by Smirnov \& Sobolev (1933), Petrashen' et al. (1950), Cagniard (1962), De Hoop (1960), etc. The formal equivalence between the plane strain and plane stress problems of elasticity prompted Kaplunov \& Nol'de (1992) to consider a generalisation of Lamb's problem, specifically the problem of instantaneous loading of an edge point for a thin semi-infinite elastic plate. Their study revealed considerable discrepancies between predictions of the plane stress equations and the corresponding exact three-dimensional theory. It was in particular shown that the plane stress solution distorts the speed of propagation of

\footnotetext{
*Present address: Department of Civil and Structural Engineering, University of Sheffield, Sir Frederick Mappin Building, Mappin Street, Sheffield S1 3JD, UK.

${ }^{\dagger}$ Corresponding author
} 
the dilatational wave. The propagating discontinuity of the dilatational potential predicted by the plane stress theory should in fact be a smooth disturbance, which we term as a quasi-front. It was also demonstrated that the principal features of the response were well reproduced by a refined long-wave low-frequency theory without having to resort to the exact equations of three-dimensional elasticity. The complicated problem of refining edge boundary conditions was avoided by using the method of matched asymptotic expansions. Specifically, the outer problem was solved for non-refined plate theory and boundary conditions. The effects of higher-order terms arising in the refined plate theory were accounted for by the inner problem, considered in the vicinity of the characteristics of hyperbolic outer problem. This approach was later successfully extended to complicated geometries and other types of media by Kaplunov \& Nol'de (1995) and Emri et al. (2001).

This paper seeks to extend the aforementioned technique to the case of pre-stressed incompressible elastic plates. The effects of pre-stress on elastic wave propagation in incompressible media are considerable, see e.g. studies on surface wave propagation by Dowaikh \& Ogden (1990) and plane wave reflection by Ogden \& Sotiropoulos (1998). When studying the dynamics of pre-stressed plates, Kaplunov et al. (2000) and Pichugin \& Rogerson (2002) demonstrated how variations in pre-stress may be used to change the type of generated quasifronts from the classical receding to advancing. They also indicated the presence of a new type of quasi-front in pre-stressed media, the bending quasi-front. This front was studied by Kaplunov \& Pichugin (2005). By introducing the simplifying assumption of bi-axial primary deformations, the extensional longwave low-frequency theory for pre-stressed incompressible media may be made formally equivalent to an analogous isotropic theory. This opportunity is used in this paper to highlight possible deviations from the far-field responses normally expected in respect of the isotropic plates.

\section{Governing equations}

\subsection{Modelling of a bi-axially pre-stressed medium}

We consider a body composed of an incompressible isotropic Green-elastic material. It is pre-stressed, which implies here that it is statically and homogeneously deformed from a natural unstressed state into a reference configuration $\mathcal{B}_{e}$. Incremental motions of the body may then be described by the following set of equations of motion

$$
B_{m i l k} u_{k, l m}-p_{t, i}=\rho \ddot{u}_{i},
$$

where comma and over-dot denote differentiation with respect to the indicated space and time variables and the summation convention is employed, see Ogden (1984). The vector function $\boldsymbol{u}$ specifies infinitesimal displacements of material points in $\mathcal{B}_{e}$ and the scalar $p_{t}$ is a time-dependant increment of the 'pressure' $p \equiv \bar{p}+p_{t}$, the reaction stress necessary to accommodate the linearised incompressibility constraint $u_{i, i}=0$. It is usually more convenient to relate the static component of pressure $\bar{p}$ to one of the principal Cauchy stresses; we therefore note that $\bar{p}=B_{2121}-B_{1212}-\bar{\sigma}_{2}$, with $\bar{\sigma}_{2}$ denoting the normal Cauchy stress at the upper layer face. It is also remarked that $\boldsymbol{B}$ is the fourth order tensor of instantaneous elastic moduli evaluated in the $\mathcal{B}_{e}$. 


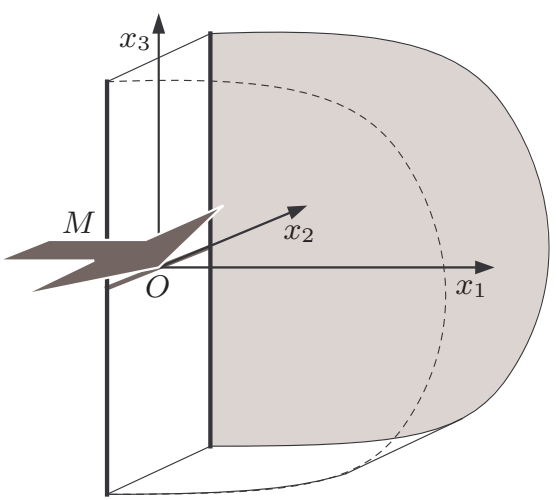

Figure 1: Semi-infinite plate subjected to an instantaneous impulse loading on the edge segment.

In order to investigate transient wave phenomena in a semi-infinite layer, it is convenient to introduce a Cartesian coordinate system with the origin in the mid-plane of the layer, as shown in Figure 1. We further assume that the principal axes of the primary homogeneous deformation coincide with the coordinate axes. When the considered primary deformation is bi-axial, within the plane of the layer, the only non-zero elastic moduli in (1) are given by

$$
\begin{gathered}
B_{1111}=B_{1133}=B_{3311}=B_{3333}, \quad B_{1122}=B_{2211}=B_{2233}=B_{3322}, \\
B_{1212}=B_{3232}, \quad B_{2121}=B_{2323}, \quad B_{1221}=B_{2112}=B_{2332}=B_{3223}, \\
B_{2222}, \quad B_{1313}=B_{3131}=-B_{1331}=-B_{3113} .
\end{gathered}
$$

The incremental equivalent of the classical free face boundary conditions may be derived from a linearised measure of incremental surface traction, which is conveniently introduced in the following form

$$
\tau_{\overline{\mathbf{n}} i}=B_{m i l k} u_{k, l} \bar{n}_{m}+\left(B_{2121}-B_{1212}-\sigma_{2}\right) u_{m, i} \bar{n}_{m}-p_{t} \bar{n}_{i},
$$

in which vector $\overline{\boldsymbol{n}}$ defines the outward unit normal to a layer surface, $\sigma_{2}$ is the Cauchy stress in $\mathcal{B}_{e}$ and summation over the repeated suffices is assumed.

\subsection{Long-wave low-frequency theory for plate extension}

It is a well known fact that for the majority of practical applications the farfield transient response of thin structures is dominated by long waves, i.e. waves whose wavelength is considerably greater than structure's characteristic dimension, see Kaplunov et al. (1998). Use of various long-wave low-frequency structural theories has therefore become a customary approach when modelling transient phenomena in structures. In the case of a semi-infinite plate, the natural choice of a small asymptotic parameter is $\eta \equiv h / L$, defined as ratio of the plate half-thickness $h$ to distance $L$ from the point of loading $O$ to an actual observation point. When $\eta \ll 1$, it is possible to use a lower-dimensional asymptotic plate theory to accurately describe the major features of the associated farfield solution. The symmetry of a plate allows further simplifications by separating the long-wave low-frequency response into its symmetric (extensional) 
and anti-symmetric (flexural) components. These correspond to the classical plate extension and bending theories, respectively. The anti-symmetric transient response of a semi-infinite pre-stressed incompressible plate subjected to an instantaneous out-of-plane edge impulse loading has already been studied by Kaplunov \& Pichugin (2005), using the asymptotic theory derived by Pichugin \& Rogerson (2002). Thus, this investigation is focused on long-wave low-frequency extensional motion, which may be described by the refined asymptotic theory ${ }^{1}$

$$
\begin{gathered}
\frac{\partial^{2} \phi}{\partial x_{1}^{2}}+\frac{\partial^{2} \phi}{\partial x_{3}^{2}}-\frac{1}{c_{d}^{2}} \frac{\partial^{2} \phi}{\partial t^{2}}+\frac{\mathcal{E} \eta^{2}}{3 c_{d}^{2}}\left(\frac{\partial^{4} \phi}{\partial x_{1}^{4}}+2 \frac{\partial^{4} \phi}{\partial x_{1}^{2} \partial x_{3}^{2}}+\frac{\partial^{4} \phi}{\partial x_{3}^{4}}\right)=0 \\
\frac{\partial^{2} \psi}{\partial x_{1}^{2}}+\frac{\partial^{2} \psi}{\partial x_{3}^{2}}-\frac{1}{c_{s}^{2}} \frac{\partial^{2} \psi}{\partial t^{2}}=0
\end{gathered}
$$

in which $\phi=\phi\left(x_{1}, x_{3}, t\right)$ and $\psi=\psi\left(x_{1}, x_{3}, t\right)$ are the usual dilatational and distortional displacement potentials implicitly defined by

$$
u_{1}=\frac{\partial \phi}{\partial x_{1}}+\frac{\partial \psi}{\partial x_{3}}, \quad u_{2}=-x_{2}\left(\frac{\partial^{2} \phi}{\partial x_{1}^{2}}+\frac{\partial^{2} \phi}{\partial x_{3}^{2}}\right), \quad u_{3}=\frac{\partial \phi}{\partial x_{3}}-\frac{\partial \psi}{\partial x_{1}},
$$

where $u_{i}=u_{1}\left(x_{1}, x_{2}, x_{3}, t\right), i \in\{1,2,3\}$, are the leading order plate displacements. The material and pre-stress parameters in (4) and (5) are given by

$$
\begin{gathered}
\rho c_{d}^{2}=B_{1111}+B_{2222}-2 B_{1122}-2 B_{1221}+2\left(B_{2121}-\sigma_{2}\right), \\
\rho c_{s}^{2}=B_{1313}, \quad \rho \mathcal{E}=\rho c_{d}^{2}-B_{1212}+\frac{\left(B_{2121}-\sigma_{2}\right)^{2}}{B_{2121}} .
\end{gathered}
$$

All quantities in (4), (5) are non-dimensional, assumed to be $O(1)$, and related to their dimensional counterparts through

$$
\hat{x}_{1}=L x_{1}, \quad \hat{x}_{3}=L x_{3}, \quad \hat{t}=L c_{0} t, \quad \hat{\phi}=L^{2} \phi, \quad \hat{\psi}=L^{2} \psi,
$$

within which $c_{0}$ is a chosen unit of speed and a hat denotes un-scaled dimensional quantities. The non-dimensional dilatational $c_{d}$ and shear $c_{s}$ wave speeds are both given in terms of $c_{0}$, so that $c_{d}=\hat{c}_{d} / c_{0}$ and $c_{s}=\hat{c}_{s} / c_{0}$. This form of rescaling allows for clearer interpretation of the physical significance of subsequent results.

Equations (4), (5) appear to be formally equivalent to the analogous longwave low-frequency models for isotropic plates. It is worth noting that the decoupling of the dilatational and distortional wave fields, evident from these equations, is only possible when the pre-stress within the plane of a plate is bi-axial. As in the isotropic case, it is possible to demonstrate that the asymptotic theory (4), (5) can only be used to describe motions whose dominant wavelength does not exceed $\eta^{-4 / 5}$, see e.g. Kaplunov \& Nol'de (1992). Also, again in parallel with isotropic theories, we observe that higher-order correction terms only enter the governing equation for the dilatational wave potential.

\footnotetext{
1 The detailed derivation of a long-wave low-frequency model for extension of a bi-axially pre-stressed plate is a laborious task and can hardly be justified within the scope of this paper A more general asymptotic model that is not limited to the requirement of bi-axial primary deformations will become the subject of a separate forthcoming publication. Procedures for the asymptotic derivation of structural models may be found in works by Kaplunov et al. (1998) or Kaplunov et al. (2000).
} 
This indicates the impossibility of a distortional quasi-front. However, possible parameter variations in the model demonstrate significant departures from the classical isotropic theory. Firstly, while the coefficient $\mathcal{E}$ of higher-order correction terms is always positive in isotropic elasticity, it may become negative in a pre-stressed medium, which in Section 3 will be demonstrated to alter the type of generated quasi-front. Secondly, the local stability considerations for isotropic media impose a requirement that $c_{d}>c_{s}$. This requirement no longer holds for a pre-stressed medium, so that it is possible to choose finitely deformed configurations where the dilatational wave will propagate behind the shear wave, which we term configurations with slow quasi-fronts. It is not only the order of arrival of the disturbances that is different in this situation; the nature of the head-wave, generated by potential's coupling at the plate edge, changes from being essentially distortional to a dilatational. This also means that the head-wave itself is to be smoothed by the higher-order correction of our plate theory, a phenomenon discussed in Section 4.

\subsection{Statement of the problem}

We are ultimately interested in the far-field response of a plate, described by the theory (4), (5), to an instantaneous impulse loading at an edge point, see Figure 1. Thus, generally we need a method of prescribing three tractions at the free edge of the plate. However, only two boundary conditions may be satisfied with equations (4), (5). We therefore choose to prescribe the two tractions, which are asymptotically leading for the considered motions, i.e. long-wave lowfrequency extensions. Specifically, the loading is applied according to

$$
\begin{gathered}
\sigma_{1} \equiv c_{d}^{2} \frac{\partial^{2} \phi}{\partial \xi_{1}^{2}}+\alpha \frac{\partial^{2} \psi}{\partial \xi_{1} \partial \xi_{3}}+\left(c_{d}^{2}-\alpha\right) \frac{\partial^{2} \phi}{\partial \xi_{3}^{2}}=-M_{n} \delta\left(\xi_{3}\right) \delta(\tau) \\
\tau_{13} \equiv \alpha \frac{\partial^{2} \phi}{\partial \xi_{1} \partial \xi_{3}}-c_{s}^{2} \frac{\partial^{2} \psi}{\partial \xi_{1}^{2}}+\left(\alpha-c_{s}^{2}\right) \frac{\partial^{2} \psi}{\partial \xi_{3}^{2}}=0
\end{gathered}
$$

in which $\delta(\cdot)$ is Dirac's delta function, $\sigma_{1}$ and $\tau_{13}$ are the normal and in-plane tangential Cauchy stresses at the plate edge, respectively, $M_{n}$ is the load magnitude and

$$
\rho \alpha=B_{2121}-B_{1221}-\sigma_{2} .
$$

As is customary for non-stationery loading problems, all functions occurring in (4), (5) and (8), (9) are to be understood in the generalised sense. Thus pure normal stress is to be applied instantaneously at the point $O$. This loading is highly idealised; such degree of localisation both in space and time is hardly realisable from the physical point of view. However, the far-field dilatational response to this localised loading will be demonstrated to be smoothed and strongly dispersive. For more realistic (less localised in space and/or time) loadings the long-wave low-frequency analysis presented in this paper will be even more relevant. We remark that the effects of an in-plane tangential loading $\left(\tau_{13} \neq 0\right)$ will not be considered in this paper.

Since $\tau_{12}$ is left unprescribed, we ought to expect the error of approximation for our boundary conditions to be $O(\eta)$. Thus, generally the refined theory (4), (5) cannot be used in conjunction with the leading order boundary conditions (8), (9). In order to circumvent this difficulty the method of matched asymptotic expansions may be applied, see e.g. Cole (1968). For an 
outer problem we use the leading order hyperbolic plate theory given by (4), (5) with the higher-order terms omitted. This may be used in conjunction with the boundary conditions (8), (9) to provide a leading order solution, which is valid everywhere except in a vicinity of hyperbolic wave fronts predicted by the leading order theory. The contribution of higher-order terms in (4) becomes significant near the dilatational wave front. In an appropriately chosen inner coordinate system these terms enter at leading order and describe the dispersive smoothing of the discontinuity propagating along a characteristic. The asymptotic matching principle may then be applied to determine the leading order solution valid in a vicinity of the dilatational quasi-front.

Validity of the described matching procedure may be justified by the fact that any higher order correction terms, entering the boundary conditions (8), (9), would only affect the second order amplitude of the outer solution, whilst the phase would remain unchanged, see Kaplunov \& Nol'de (1992). The leading order far-field solution for a quasi-front is therefore essentially independent of the boundary layers, predicted by the full three-dimensional theory near the plate egde. This observation is by no means implying that these boundary layers may be ignored when studying disturbance propagating near the edge. However, the far-field response near the edge is usually dominated by the surface (edge) wave, which makes solutions, describing any other features of the wave field near plate's edge, less useful.

\section{Solution for a fast quasi-front}

\subsection{Outer problem}

In solving the outer problem we closely follow the technique developed for the isotropic plane strain problem by Petrashen' et al. (1950). Their method of contour integration is chosen because it offers clear physical interpretation for the behaviour of obtained solutions. The leading order governing equations corresponding to (4), (5) are considered in the form

$$
\begin{aligned}
& c_{d}^{2} \frac{\partial^{2} \phi_{\text {out }}}{\partial x_{1}^{2}}+c_{d}^{2} \frac{\partial^{2} \phi_{\text {out }}}{\partial x_{3}^{2}}-\frac{\partial^{2} \phi_{\text {out }}}{\partial t^{2}}=0, \\
& c_{s}^{2} \frac{\partial^{2} \psi_{\text {out }}}{\partial x_{1}^{2}}+c_{s}^{2} \frac{\partial^{2} \psi_{\text {out }}}{\partial x_{3}^{2}}-\frac{\partial^{2} \psi_{\text {out }}}{\partial t^{2}}=0,
\end{aligned}
$$

and are to be solved subject to the boundary conditions (8), (9) and zero initial conditions, i.e. $\phi_{\text {out }}=\dot{\phi}_{\text {out }}=\psi_{\text {out }}=\dot{\psi}_{\text {out }}=0$, when time $t=0$. The formal transform solution may then be derived by subsequent Fourier and Laplace transforms of the leading order governing equations with respect to time $t$ and space variable $x_{3}$, respectively. The resulting system of the ordinary differential equations may be written as

$$
\begin{aligned}
& c_{d}^{2} \frac{\partial^{2} \phi_{\mathrm{out}}^{\mathrm{FL}}}{\partial x_{1}^{2}}-\left(c_{d}^{2} k_{3}^{2}+s^{2}\right) \phi_{\mathrm{out}}^{\mathrm{FL}}=0, \\
& c_{s}^{2} \frac{\partial^{2} \psi_{\text {out }}^{\mathrm{FL}}}{\partial x_{1}^{2}}-\left(c_{s}^{2} k_{3}^{2}+s^{2}\right) \psi_{\mathrm{out}}^{\mathrm{FL}}=0,
\end{aligned}
$$


subject to the boundary conditions

$$
\begin{aligned}
& c_{d}^{2} \frac{\partial^{2} \phi_{\text {out }}^{\mathrm{FL}}}{\partial x_{1}^{2}}+\mathrm{i} \alpha k_{3} \frac{\partial \psi_{\text {out }}^{\mathrm{FL}}}{\partial x_{1}}-k_{3}^{2}\left(c_{d}^{2}-\alpha\right) \phi_{\text {out }}^{\mathrm{FL}}=-M_{n}, \\
& \mathrm{i} \alpha k_{3} \frac{\partial \phi_{\mathrm{out}}^{\mathrm{FL}}}{\partial x_{1}}-c_{s}^{2} \frac{\partial^{2} \psi_{\mathrm{out}}^{\mathrm{FL}}}{\partial x_{1}^{2}}-k_{3}^{2}\left(\alpha-c_{s}^{2}\right) \psi_{\mathrm{out}}^{\mathrm{FL}}=0
\end{aligned}
$$

where zero initial conditions ensure the existance of the inverse Laplace transform. General solutions of the boundary value problem (13)-(16) that decay as $s \rightarrow \infty$ and therefore guarantee the necessary Laplace transform behaviour are given by

$$
\begin{aligned}
& \phi_{\text {out }}^{\mathrm{FL}}\left(x_{1}, k_{3}, s\right)=C_{\phi}\left(k_{3}, s\right) \exp \left(-\frac{x_{1}}{c_{d}} \sqrt{c_{d}^{2} k_{3}^{2}+s^{2}}\right), \\
& \psi_{\text {out }}^{\mathrm{FL}}\left(x_{1}, k_{3}, s\right)=C_{\psi}\left(k_{3}, s\right) \exp \left(-\frac{x_{1}}{c_{s}} \sqrt{c_{s}^{2} k_{3}^{2}+s^{2}}\right),
\end{aligned}
$$

within which

$$
\begin{gathered}
C_{\phi}\left(k_{3}, s\right)=-\frac{c_{d} c_{s}\left(\alpha k_{3}^{2}+s^{2}\right) M_{n}}{R\left(k_{3}, s\right)}, \quad C_{\psi}\left(k_{3}, s\right)=\frac{\mathrm{i} c_{s} \alpha k_{3} \sqrt{c_{d}^{2} k_{3}^{2}+s^{2}} M_{n}}{R\left(k_{3}, s\right)}, \\
R\left(k_{3}, s\right)=c_{d} c_{s}\left(\alpha k_{3}^{2}+s^{2}\right)^{2}-\alpha^{2} k_{3}^{2} \sqrt{c_{s}^{2} k_{3}^{2}+s^{2}} \sqrt{c_{d}^{2} k_{3}^{2}+s^{2}}
\end{gathered}
$$

Using the symmetry of the solutions (17), (18) with respect to $k_{3}$ we represent the formal transform solution of the outer problem in the following form

$$
\begin{aligned}
& \phi_{\text {out }}\left(x_{1}, x_{3}, t\right)=\frac{\mathrm{i} c_{s} c_{d} M_{n}}{2 \pi^{2}} \times \\
& \int_{0}^{\infty} \int_{\sigma-\mathrm{i} \infty}^{\sigma+\mathrm{i} \infty} \frac{\left(\alpha k_{3}^{2}+s^{2}\right)}{R\left(k_{3}, s\right)} \exp \left(s t-\frac{x_{1}}{c_{d}} \sqrt{c_{d}^{2} k_{3}^{2}+s^{2}}\right) d s \cos \left(k_{3} x_{3}\right) d k_{3}, \\
& \psi_{\text {out }}\left(x_{1}, x_{3}, t\right)=\frac{\mathrm{i} c_{s} \alpha M_{n}}{2 \pi^{2}} \times \\
& \int_{0}^{\infty} \int_{\sigma-\mathrm{i} \infty}^{\sigma+\mathrm{i} \infty} \frac{\sqrt{c_{d}^{2} k_{3}^{2}+s^{2}}}{R\left(k_{3}, s\right)} \exp \left(s t-\frac{x_{1}}{c_{s}} \sqrt{c_{s}^{2} k_{3}^{2}+s^{2}}\right) d s k_{3} \sin \left(k_{3} x_{3}\right) d k_{3} .
\end{aligned}
$$

Equations (19) and (20) provide an exact solution to the outer problem. It is complicated and computationally inefficient. However, this form of the solution permits detailed asymptotic analysis for the principal features of the generated disturbance, see Petrashen' et al. (1950). Since we are interested in studying the effects of the higher-order terms in equation (4), only the parts of response featuring rapid variations of the dilatational potential $\phi$ are of primary interest. With this in mind, we concentrate our further analysis on (19), omitting all results for the distortional potential $\psi$ for the sake of brevity.

For waves propagating in linear isotropic elastic media it is always the case that $c_{d}>c_{s}$, meaning that the dilatational wave front, which is to be smoothed by the higher-order plate theory correction, propagates faster than its distortional counterpart. The situation is therefore termed here the case of fast quasifront. A solution in the vicinity of a wave front is dominated by short waves $\left(k_{3} \gg 1\right)$, which enables us to use the steepest descent method to evaluate the 


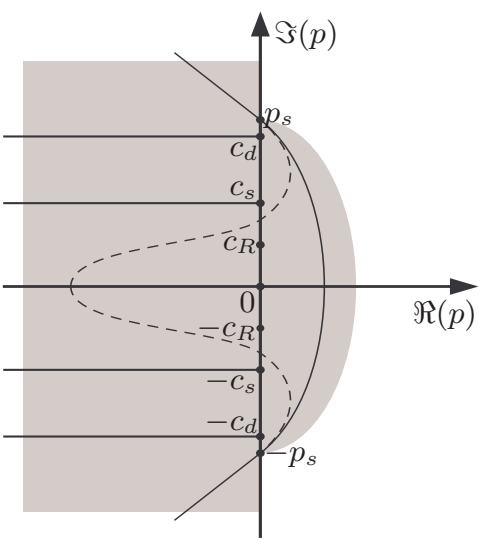

(a)

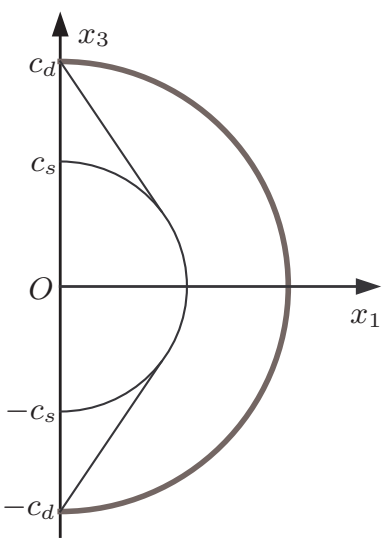

(b)

Figure 2: (a) The phase plane for the Laplace transform parameter $p$ when $c_{d}>c_{s} . \pm \mathrm{i} p_{s}$ are the saddle points, $\pm \mathrm{i} c_{d}$ and $\pm \mathrm{i} c_{s}$ the branch points, $\pm \mathrm{i} c_{R}$ and 0 the poles of the Mellin integral within $(19)$ and $\Re(f(p)) \leqslant 0$ in the shaded area. (b) The corresponding wave front configuration at the time instant $t=1$, where the bold line denotes dilatational disturbance.

Mellin integral within (19). First, it is convenient to introduce $p \equiv s / k_{3}$ in (19) so that

$$
\begin{aligned}
& \phi_{\mathrm{out}}^{\mathrm{F}}\left(x_{1}, k_{3}, t\right)=\frac{\mathrm{i} c_{d} c_{s} M_{n}}{2 \pi^{2} k_{3}} \times \\
& \int_{\sigma^{\prime}-\mathrm{i} \infty}^{\sigma^{\prime}+\mathrm{i} \infty} \frac{\left(\alpha+p^{2}\right)}{R(p)} \exp \left(k_{3}\left\{p t-\frac{x_{1}}{c_{d}} \sqrt{c_{d}^{2}+p^{2}}\right\}\right) d p \\
& R(p)=c_{d} c_{s}\left(\alpha+p^{2}\right)^{2}-\alpha^{2} \sqrt{c_{s}^{2}+p^{2}} \sqrt{c_{d}^{2}+p^{2}}
\end{aligned}
$$

Square root values are fixed by requiring that $\arg \sqrt{c_{d}^{2}+p^{2}}=\arg \sqrt{c_{s}^{2}+p^{2}}=0$ when $p>0$. Branch cuts are introduced from the points $\pm \mathrm{i} c_{d}$ and $\pm \mathrm{i} c_{s}$ parallel to the negative part of the real axis, see the phase plane depicted in Figure 2(a). In order to ensure that the transient disturbance decays as it propagates away from the plate edge, we need the real part of the phase function

$$
f(p) \equiv p t-\frac{x_{1}}{c_{d}} \sqrt{c_{d}^{2}+p^{2}}
$$

to be negative along the integration contour. This occurs when $\Re(p)<0$, as well as when $p$ is within the ellipse

$$
\frac{\Re(p)^{2}}{x_{1}^{2}}+\frac{\Im(p)^{2}}{c_{d}^{2} t^{2}}=\frac{c_{d}^{2}}{c_{d}^{2} t^{2}-x_{1}^{2}},
$$

see the shaded area within Figure 2(a). The saddle points of $f(p)$ are given by $p_{s}= \pm \mathrm{i} c_{d} / \zeta$, where $\zeta^{2} \equiv 1-x_{1}^{2} / c_{d}^{2} t^{2}$, hence behind the dilatational wave front $\left|p_{s}\right|>\left|c_{d}\right|$ (both potentials must be equal to zero before the arrival of wave fronts). The integration contour may therefore be chosen to go through the saddle points while remaining in the shaded area, as shown in Figure 2(a) 
by the thin solid curve. The corresponding leading order steepest descent approximation of (21) has the form

$$
\phi_{\text {out }}^{\mathrm{F}}\left(x_{1}, k_{3}, t\right)=\frac{M_{n} \sqrt{2 c_{d} \zeta} c_{s} x_{1}\left(c_{d}^{2}-\alpha \zeta^{2}\right) \Re\left(\exp \left(\mathrm{i}\left\{k_{3} c_{d} t \zeta+\frac{\pi}{4}\right\}\right)\right)}{\sqrt{\pi^{2} t^{3} k_{3}^{3}}\left(c_{s} c_{d}\left(c_{d}^{2}-\alpha \zeta^{2}\right)^{2}+\alpha^{2} \zeta^{2} \frac{x_{1}}{t} \sqrt{c_{d}^{2}-c_{s}^{2} \zeta^{2}}\right)}+O\left(k_{3}^{-1}\right),
$$

see Erdélyi (1987). The approximation (24) is only valid provided $k_{3} \gg \zeta^{-1}$, which in particular means that (24) is invalid at the tip of the wave front. This does not indicate a non-uniformity of the behaviour of solution (21), but only a non-uniformity of the approximation itself; for account of a similar problem see Kaplunov \& Pichugin (2005).

It is worth remarking that although the contour that we have chosen satisfies all of the necessary requirements for the steepest descent method, approximation (24) may become quite inaccurate away from the dilatational wave front, especially for relatively small values of $k_{3}$. The results may be considerably improved by moving the integration contour across the $\Im(p)$ axis as shown in Figure 2(a) by the dashed line. The contributions from the Rayleigh poles ${ }^{2} \pm \mathrm{i} c_{R}$ and the double static solution pole at 0 will then enter the approximation, see Petrashen' et al. (1950). However, for our purposes the outer solution (24) is sufficient.

\subsection{Inner solution}

The contribution of the higher-order terms in the refined equation (4) is particularly significant in the vicinity of the dilatational wave front predicted by (11). This is best accounted for by introducing an appropriate system of coordinates associated with the wave front. Thus, we introduce inner coordinates defined implicitly by

$$
x_{1}=c_{d} \tau \cos \theta, \quad x_{3}=c_{d}\left(\tau \sin \theta+\frac{\eta^{\varepsilon} \xi}{\sin \theta}\right), \quad t=\tau .
$$

This coordinate system is very similar to the one developed by Kaplunov \& Nol'de (1995). An arbitrary point $M$ is characterised by its distance $\xi$ from the dilatational wave front along $O x_{3}$ and the angle $\theta$ between $O x_{1}$ and the point where $\xi=0$, see Figure 3 . The scaling parameter $\eta^{\varepsilon}$ 'magnifies' the vicinity of the wave front, so that the parameter $\varepsilon$ serves as a logarithmic measure of the quasi-front width, which is $O\left(\eta^{-\varepsilon}\right)$. It may then be established that the fourth order derivative terms in (11) are balanced with the second order derivative terms when $\varepsilon=2 / 3$, with an appropriate leading order inner governing equation having the form

$$
\frac{\phi_{\text {in }}}{\tau}+2 \frac{\partial \phi_{\text {in }}}{\partial \tau}+\frac{\mathcal{E}}{3 c_{d}^{4}} \frac{\partial^{3} \phi_{\text {in }}}{\partial \xi^{3}}=0,
$$

in which $\phi_{\text {in }} \equiv \phi_{\text {in }}(\xi, \theta, \tau)$. This one-dimensional equation is easy to solve using the Fourier transform with respect to $\xi$. The general transform solution of the

\footnotetext{
2 In the context of the dynamics for semi-infinite plates the Rayleigh-type denominator $R(p)$ and the associated poles characterise the long-wave low-frequency component of an edge wave, i.e. the wave confined to the plate edge and exponentially decaying away from it.
} 


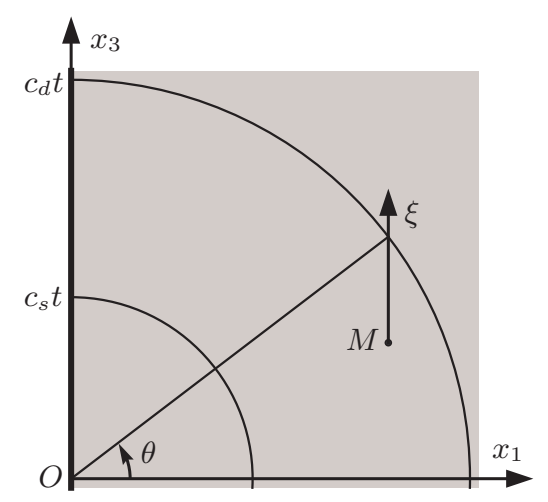

Figure 3: The system of inner coordinates for the dilatational quasi-front.

inner problem is then given by

$$
\phi_{\text {in }}^{\mathrm{F}}(\xi, \theta, \tau)=\frac{C_{\mathrm{in}}\left(k_{\xi}, \theta\right)}{\sqrt{\tau}} \exp \left(\mathrm{i} \frac{\mathcal{E} \tau k_{\xi}^{3}}{6 c_{d}^{4}}\right),
$$

where $k_{\xi}$ is the wave number associated with $\xi$.

\subsection{Asymptotic matching procedure}

The unknown function $C_{\text {in }}\left(k_{\xi}, \theta\right)$ in $(27)$ is to be determined by the method of matched asymptotic expansions. Here we use a simple matching principle of the following form: an inner limit of the outer solution must be equal to an outer limit of the inner solution. We begin by substituting the outer variables, defined by (25), into the inner transform solution (27) and taking the asymptotic limit as $\eta \rightarrow 0$. The outer limit of (27) may then conveniently be given in terms of inner variables as

$$
\phi_{\mathrm{in}}^{\mathrm{F}}(\xi, \theta, \tau) \sim \frac{C_{\mathrm{in}}\left(k_{\xi}, \theta\right)}{\sqrt{\tau}} .
$$

The inner limit of the steepest descent approximation (24) of the outer solution is found using the similar procedure. In terms of the inner variables it has the following form

$$
\phi_{\text {out }}(\xi, \theta, \tau) \sim \frac{M_{n} c_{s} c_{d} \eta^{1 / 3}}{\sqrt{2 \pi^{3} \tau} R_{\text {in }}(\theta)} \int_{0}^{\infty} \sin \left(\xi k_{\xi}+\frac{\pi}{4}\right) \frac{d k_{\xi}}{\sqrt{k_{\xi}^{3}}}, \quad \eta \rightarrow 0
$$

where we have defined

$$
R_{\mathrm{in}}(\theta)=\frac{c_{s}\left(c_{d}^{2}-\alpha \sin ^{2} \theta\right)}{\cos \theta}+\frac{\alpha^{2} \sin ^{2} \theta \sqrt{c_{d}^{2}-c_{s}^{2} \sin ^{2} \theta}}{\left(c_{d}^{2}-\alpha \sin ^{2} \theta\right)} .
$$

The divergent integral in (29) must only be understood as a formal statement of the fact that within the vicinity of the wave front $\phi_{\text {out }}(\xi, \theta, \tau)$ is asymptotically equal to the high frequency contributions of the Fourier transform. The contribution of the pole $k_{\xi}=0$ must be ignored. The divergence of this integral is caused by the (essentially local) change of variables $p \equiv s / k_{3}$ performed in (21). 
The matching procedure is implemented by re-casting (29) in the form of a double-sided Fourier transform integral and then comparing the result with (28). The resulting function $C_{\text {in }}\left(k_{\xi}, \theta\right)$ may be written as

$$
C_{\text {in }}\left(k_{\xi}, \theta\right)=\frac{(1+\mathrm{i}) M_{n} c_{s} c_{d} \eta^{1 / 3}}{2 \sqrt{\pi k_{\xi}^{3}} R_{\text {in }}(\theta)} .
$$

By inserting (31) into the inner solution (27), and using the symmetry of the resulting inverse Fourier transform integral, the quasi-front asymptotic representation for the dilatational wave potential may be given in the following form

$$
\phi_{\text {in }}\left(\xi^{*}, \theta, \tau\right)=\frac{M_{n} c_{s} c_{d} \sqrt{|\sin \theta|}}{\sqrt{2 \pi^{3} \tau} R_{\text {in }}(\theta)} \int_{0}^{\infty} \sin \left(\zeta \xi^{*}-\frac{\mathcal{E} \tau \zeta^{3} \eta^{2}}{6 c_{d}^{4}\left|\sin ^{3} \theta\right|}+\frac{\pi}{4}\right) \frac{d \zeta}{\sqrt{\zeta^{3}}},
$$

within which $\xi^{*}=\xi \eta^{2 / 3} / \sin \theta$. This in particular means that

$$
\xi^{*}=\frac{1}{c_{d}}\left(x_{3}-\sqrt{c_{d}^{2} \tau^{2}-x_{1}^{2}}\right)
$$

see (25). By combining this relationship with the high wave number approximation (32) we arrive at the following asymptotic expressions for the in-plane displacements in terms of the $\left(\xi^{*}, \theta, \tau\right)$ coordinate system

$$
\begin{aligned}
& u_{1}=\frac{M_{n} c_{s} c_{d}^{2} \sqrt{\tau^{3}} \cos \theta}{\sqrt{2 \pi^{3}} R_{\operatorname{in}}(\theta) \sqrt{|\sin \theta|}} \Phi\left(\xi^{*},-\frac{\mathcal{E} \tau \eta^{2}}{6 c_{d}^{4}\left|\sin ^{3} \theta\right|}\right), \\
& u_{3}=\frac{M_{n} c_{s} \operatorname{sgn} \theta \sqrt{|\sin \theta|}}{\sqrt{2 \pi^{3} \tau} R_{\text {in }}(\theta)} \Phi\left(\xi^{*},-\frac{\mathcal{E} \tau \eta^{2}}{6 c_{d}^{4}\left|\sin ^{3} \theta\right|}\right),
\end{aligned}
$$

in which we introduced the following special function

$$
\Phi(x, \varepsilon)=\int_{0}^{\infty} \cos \left(x k+\varepsilon k^{3}+\frac{\pi}{4}\right) \frac{d k}{\sqrt{k}} \equiv 2 \int_{0}^{\infty} \cos \left(x t^{2}+\varepsilon t^{6}+\frac{\pi}{4}\right) d t,
$$

related to the one encountered by Kaplunov \& Nol'de (1995). The general properties and asymptotic behaviour of this function were previously investigated by Kaplunov \& Pichugin (2005).

It is a good time now to discuss the range of applicability of the solution (34), (35). We already mentioned in Section 2.2, that our asymptotic plate theory can only be used for motions dominated by wave numbers $k \ll \eta^{-4 / 5}$. On the other hand, the steepest descent approximation of the outer solution (24) is valid only provided $k \gg \zeta^{-1}=|\sin \theta|^{-1}$. This means that generally we need to ensure that $|\sin \theta|^{-1} \ll k \ll \eta^{-4 / 5}$. Let us now estimate the area, where the solution (34), (35) is valid for times $\tau \sim 1$. Bounds on $\theta$ may be determined from the second order terms, omitted from the leading order inner governing equation (26), which is valid provided $|\sin \theta| \gg \eta^{2 / 3}$. There can be no upper bound for $\theta$ as within the realm of two-dimensional theory the solution is valid even at the plate edge. A typical way to deal with this difficulty would be to assume that the three-dimensional edge boundary layer decays within a plate thickness off the edge, so that $\cos \theta>\eta$. At last, it is necessary to 
determine the range of $\xi^{*}$ where our approximation may be used. It is easy to see from the definition (36) of function $\Phi(x, \varepsilon)$ that the effect of the higher-order dispersion correction terms is related to the introduction of a stationery point $k \sim \sqrt{-\mathcal{E} \xi^{*}} / \eta$, which is essentially a wave number dominating our solution in the vicinity of a wave front. Thus, by relating this wave number to the applicability ranges of the plate theory and steepest descent approximations, we conclude that $\eta^{2}|\sin \theta| \ll-\mathcal{E} \xi^{*} \ll \eta^{2 / 5}\left|\sin ^{3} \theta\right|$. Depending on the particular sign of $\mathcal{E}$, this indicates that the smoothing effect of the higher-order theory is only apparent at one side of the wave front.

The obtained asymptotic solution is not valid along the quasi-front uniformly. Specifically, the asymptotic solution fails at the tip of the quasi-front, which is caused by the failure of the steepest descent approximation (24) when $k_{3} \sim 1 / \sin \theta$, as well as singularity inherent within the inner coordinate system (25). It is worth noting that both of these issues may be dealt with. The uniform approximation for the outer solution near the wave front may, for example, be obtained using Hankel transform followed by the steepest descent approximation, see Ogurtsov \& Petrashen' (1951). The system of inner coordinates may also easily be modified to avoid the $\operatorname{singularity}$ at $\sin \theta=0$. Note that the governing equation for the inner problem would not be affected. However, these modifications would greatly complicate the matching procedure.

\section{Solution for a head-wave quasi-front}

\subsection{Outer solution}

Before discussing an approximation for a smoothed head-wave, let us study some implications of the situation when $c_{s}>c_{d}$, i.e. when the shear wave speed is higher than dilatational wave speed. It is worth reiterating that this can never happen for unstressed isotropic elastic materials, as it requires $\lambda<-\mu$ in terms of the Lamé modules. The outer transform solution (21) remains valid in this case. But the saddle points $p_{s}= \pm \mathrm{i} c_{d} / \zeta$ of the associated Laplace transform integral may now cross the branch points $\pm \mathrm{i} c_{s}$. This means that the integration contour used to obtain the steepest descent approximation (24), shown in Figure 2(a), generally has to be continued around the branch cuts from $\pm \mathrm{i} c_{s}$, as it is pictured in Figure 4(a). The saddle points coincide with branch points when $c_{s} x_{1}=c_{d} c_{\Delta} t$, within which we introduced $c_{\Delta}^{2}=c_{s}^{2}-$ $c_{d}^{2}$. When $c_{s} x_{1}>c_{d} c_{\Delta} t$, the steepest descent approximation (24) is valid, but not particularly useful because it fails for small $x_{3}$. When $c_{s} x_{1}<c_{d} c_{\Delta} t$, the dilatational wave potential consists of contributions associated with the saddle points $p_{s}$ and the branch cuts from $\pm \mathrm{i} c_{s}$, see Ogurtsov \& Petrashen' (1951). The saddle point contributions asymptotically dominate the outer solution in the vicinity of dilatational wave front, whereas the branch cut contributions dominate the outer solution in the vicinity of the head-wave, see Figure 4(b).

For the outer solution we only construct a high wave number asymptotic, which is valid within the vicinity of the head-wave. This is done by assuming that contributions of the branch cuts from $\pm \mathrm{i} c_{s}$ asymptotically dominate the saddle point contributions. An asymptotic approximation of this type for outer 


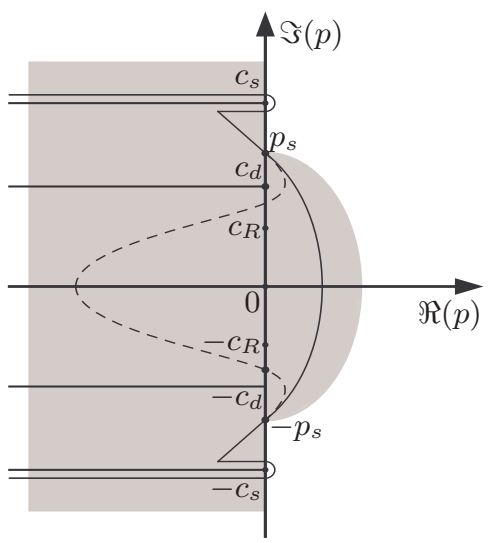

(a)

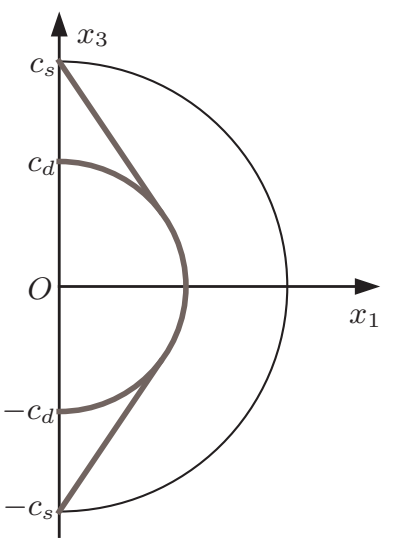

(b)

Figure 4: (a) The phase plane for the Laplace transform parameter $p$ when $c_{d}<c_{s} . \pm \mathrm{i} p_{s}$ are the saddle points, $\pm \mathrm{i} c_{d}$ and $\pm \mathrm{i} c_{s}$ the branch points, $\pm \mathrm{i} c_{R}$ and 0 the poles of the Mellin integral within (19) and $\Re(f(p)) \leqslant 0$ in the shaded area. (b) The corresponding wave front configuration at the time instant $t=1$, where the bold line denotes dilatational disturbance.

solution transform may be given by

$$
\phi_{\text {out }}^{\mathrm{F}}\left(x_{1}, k_{3}, t\right)=\frac{M_{n} \sqrt{2 c_{d} c_{\Delta}^{5}} \alpha^{2} \sin \left(\frac{k_{3}}{c_{d}}\left(c_{d} c_{s} t-c_{\Delta} x_{1}\right)+\frac{\pi}{4}\right)}{\sqrt{c_{s} \pi^{3} k_{3}^{5}\left(c_{d} c_{\Delta} t-c_{s} x_{1}\right)^{3}}\left(\alpha-c_{s}^{2}\right)^{3}}+O\left(k_{3}^{-3}\right) .
$$

Approximation (37) is obviously not valid near the point where dilatational and head-wave fronts touch. The techniques of uniform steepest descent approximation would have to be used in this case, as in the limit it corresponds to integrals in which saddle points coincide with branch cut origins.

\subsection{Inner solution}

In order to produce an appropriate inner expansion, a better knowledge of the head-wave geometry is necessary. A first quarter touching point between the dilatational front and the head-wave is shown as point $A$ in the Figure 5. It corresponds to the situation when the saddle points of the Laplace integral within (21) coincide with $\pm \mathrm{i} c_{s}$. The coordinates of $A$ are therefore given by $\left(c_{d} c_{\Delta} t / c_{s}, c_{d}^{2} t / c_{s}\right)$. A further point of the head-wave is conveniently given by $\left(0, c_{s} t\right)$. This information is sufficient to introduce a new Cartesian system of coordinates $A \xi \chi$ with the origin $A$ and the axes $\xi$ orthogonal to the head-wave and $\chi$ tangential to it, see Figure 5. Again, as in Section 3, we use the idea of magnifying the vicinity of the head-wave and introduce an inner coordinate system of the following form

$$
\begin{aligned}
& c_{s} x_{1}=\eta^{\varepsilon} c_{\Delta} \xi-c_{d} \chi+c_{d} c_{\Delta} \tau, \\
& c_{s} x_{3}=\eta^{\varepsilon} c_{d} \xi+c_{\Delta} \chi+c_{d}^{2} \tau, \quad t=\tau,
\end{aligned}
$$

where $\varepsilon$ serves as a logarithmic measure of the quasi-front width. 


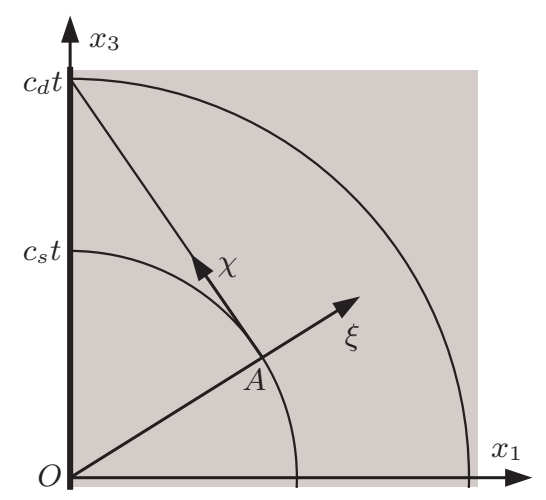

Figure 5: The system of inner coordinates for the dilatational head-wave.

By switching to the system of inner coordinates (38)-(39), and balancing the leading order dispersion terms with time-dependent terms, we are able to conclude that the width of the quasi-front in this case remains unchanged and $\varepsilon=2 / 3$. The corresponding leading order governing equation, valid within the vicinity of the head-wave, may be written as

$$
2 \frac{\partial \phi_{\text {in }}}{\partial \tau}+\frac{\mathcal{E}}{3 c_{d}} \frac{\partial^{3} \phi_{\text {in }}}{\partial \xi^{3}}=0 .
$$

The general solution of this equation may again be determined by using a Fourier transform with respect to the inner space coordinate $\xi$ (with corresponding wave number $k_{\xi}$ ) and given by

$$
\phi_{\text {in }}^{\mathrm{F}}(\xi, \tau)=C_{\mathrm{in}}\left(k_{\xi}\right) \exp \left(\mathrm{i} \frac{\mathcal{E} \tau k_{\xi}^{3}}{6 c_{d}}\right) .
$$

The governing equation (40) is well known and usually arises in plane strain problems, see e.g. Kaplunov et al. (2000). Typically, this equation is used in plane strain problems to describe configuration of the Airy quasi-front that is often given in the form of an integral of the Airy function $\operatorname{Ai}(\cdot)$. However, the next section demonstrates that the configuration of the resulting disturbance is different in the present case.

\subsection{Asymptotic matching procedure}

The outer limit of the inner expansion (37) is determined by the same procedure as used in Section 3 and may be very simply written as

$$
\phi_{\mathrm{in}}^{\mathrm{F}}(\xi, \tau) \sim C_{\mathrm{in}}\left(k_{\xi}\right), \quad \eta \rightarrow 0,
$$

where the function $C_{\text {in }}\left(k_{\xi}\right)$ may of course depend on $\chi$ as well. Its second argument is omitted because the governing equation (40) is independent of $\chi$. The corresponding inner limit of the outer expansion (37) is obtained by switching to the coordinate system (39) and taking the limit as $\eta \rightarrow 0$. By averaging over the rapidly oscillating terms, the resulting expansion takes the 
form

$$
\phi_{\mathrm{out}}^{\mathrm{F}}(\xi, \tau) \sim \frac{M_{n}(1+\mathrm{i}) \alpha^{2} c_{s} c_{\Delta}^{5 / 2} \eta}{4 \sqrt{\pi^{3} \chi^{3} c_{d}^{5} k_{\xi}^{5}}\left(c_{s}^{2}-\alpha\right)^{3}}, \quad \eta \rightarrow 0 .
$$

This high wave number approximation is similar in its form to expansion (29), however the higher degree of the wave number in the denominator indicates that the intensity of the head-wave disturbance is lower (which is obviously not true in the vicinity of the point where the head-wave touches the dilatational wave front). Expansion (42) is now matched with (43) to determine a particular value of $C_{\text {in }}\left(k_{\xi}\right)$ that does not need to be written down explicitly. Since the intensity of the head-wave quasi-front is lower, the configuration of the quasi-front is best presented in terms of stresses, not displacements. The appropriate asymptotics may be given by

$$
\begin{aligned}
\sigma_{1} & \sim \frac{M_{n} \alpha^{2} c_{\Delta}^{5 / 2}}{\sqrt{2 c_{s} \pi^{3} \chi^{3}} c_{d}\left(\alpha-c_{s}^{2}\right)^{2}} \Phi\left(\frac{c_{s}^{2}}{c_{d}^{2}} \xi^{*}, \frac{\mathcal{E} \tau c_{s}^{3} \eta^{2}}{6 c_{d}^{4}}\right), \\
\tau_{13} & \sim \frac{M_{n} \alpha^{3} c_{\Delta}^{7 / 2}}{\sqrt{2 c_{s} \pi^{3} \chi^{3}} c_{d}^{2}\left(c_{s}^{2}-\alpha\right)^{3}} \Phi\left(\frac{c_{s}^{2}}{c_{d}^{2}} \xi^{*}, \frac{\mathcal{E} \tau c_{s}^{3} \eta^{2}}{6 c_{d}^{4}}\right) .
\end{aligned}
$$

The structure of the obtained solutions is generally similar to the known solutions for two-dimensional quasi-fronts and is described by the same special function $\Phi(\cdot)$. The only principal difference is the fact that the type of headwave quasi-front, determined by the sign of the second parameter of $\Phi(\cdot)$, see Kaplunov \& Pichugin (2005), is always opposite to the type of dilatational quasi-front.

\section{Conclusion}

A presence of dilatational head-wave quasi-fronts is demonstrated for a prestressed incompressible elastic plate in plane stress. Although the dilatational head-waves were previously studied in the context of anisotropic media, see Musgrave \& Payton (1981), the associated aspects of the plane stress problem and influence of its higher-order correction are the principal results of this paper. Far-field asymptotic solutions, valid for the vicinity of quasi-fronts, are presented in a simple and computationally efficient form. An analysis of the model integrals, occurring in the solutions, is performed in Kaplunov \& Pichugin (2005). The obtained asymptotics are not uniform, however their asymptotic ranges of applicability are known.

Directions for further research involve investigation of the practicalities of application of uniform asymptotics to problems in structural dynamics. A deeper investigation of the matching procedure is also of particular interest, as it would shed more light on the physics of quasi-fronts in structures and the precise nature of the associated dispersion.

\section{Acknowledgement}

This research was supported by an EPSRC grant, GR/R53692/01, which the authors gratefully acknowledge. 


\section{References}

Cagniard, L. (1962) Reflection and Refraction of Progressive Seismic Waves. New York: McGraw-Hill.

Cole, J. D. (1968) Perturbation Methods in Applied Mathematics. Waltham MA, Toronto, London: Blaisdell Publishing Co.

De Hoop, A. T. (1960) A modification of Cagniard's method for solving seismic pulse problems. Appl. Sci. Res., Sect. B, 8, pp. 349-356.

Dowaikh, M. A. \& OGDEn, R. W. (1990) On surface waves and deformations in a pre-stressed incompressible elastic solid. IMA Jl. Appl. Math., 44, pp. 261-284.

Emri, I., Kaplunov, J. D. \& Nolde, E. V. (2001) Analysis of transient waves in thin structures utilising matched asymptotic expansions. Acta Mechanica, 149, pp. 55-68.

ERDÉlYI, A. (1987) Asymptotic Expansions. New York: Dover Publications Inc.

Kaplunov, J. D. \& Pichugin, A. V. (2005) A bending quasi-front generated by an instantaneous impulse loading at the edge of a semi-infinite pre-stressed incompressible elastic plate. Jl. Mech. Phys. Solids, 53, pp. 1079-1098.

Kaplunov, J. D., Kossovich, L. Y. \& Nolde, E. V. (1998) Dynamics of Thin Walled Elastic Bodies. New York: Academic Press.

Kaplunov, J. D., Nolde, E. V. \& Rogerson, G. A. (2000) A low frequency model for dynamic motion in pre-stressed incompressible elastic structures. Proc. R. Soc. Lond., Ser. A, 456, pp. 2589-2610.

Kaplunov, Y. D. \& Nol'DE, E. V. (1992) Lamb problem for a generalized plane stress state. Sov. Phys. Dokl., 37, pp. 88-90. Transl. from Dokl. Akad. Nauk, 322, pp. 1043-1047.

Kaplunov, Y. D. \& Nol'De, Y. V. (1995) A quasifront in the problem of the action of an instantaneous point impulse at the edge of a conical shell. J. Appl. Maths Mechs, 59, pp. 773-780. Transl. from Prikl. Mat. Mekh., 59, pp. 803-811.

LAmB, H. (1904) On the propagation of tremors over the surface of an elastic solid. Phil. Trans. Roy. Soc., Ser. A, 203, pp. 1-42.

Musgrave, M. J. P. \& Payton, R. G. (1981) Head wave contributions to elastic wave fields in a transversely isotropic half-space. Q. Jl. Mech. Appl. Math., 34, pp. 235-250.

Ogden, R. W. (1984) Non-linear Elastic Deformations. New York: Ellis Horwood.

Ogden, R. W. \& Sotiropoulos, D. A. (1998) Reflection of plane waves from the boundary of a pre-stressed compressible elastic half-space. IMA Jl. Appl. Math., 61, pp. 61-90. 
Ogurtsov, K. I. \& Petrashen', G. I. (1951) Dynamic problems for an elastic half-space in the case of an axial symmetry. Uchenie Zapiski LGU, Ser. Matem. Nauk, 149 (24), pp. 3-117. In Russian.

Petrashen', G. I., Marchuk, G. I. \& Ogurtsov, K. I. (1950) On Lamb's problem for a half-space. Uchenie Zapiski LGU, Ser. Matem. Nauk, 135 (21), pp. 71-118. In Russian.

Pichugin, A. V. \& Rogerson, G. A. (2002) An asymptotic membrane-like theory for long wave motion in a pre-stressed elastic plate. Proc. R. Soc. Lond., Ser. A, 458, pp. 1447-1468.

Smirnov, V. I. \& Sobolev, S. L. (1933) On application of the new method to investigation of elastic vibrations in axially-symmetric spaces. Tr. Seismol. Inst. AN SSSR, 29, pp. 43-51. In Russian. 